\title{
Primary $\gamma$-ray intensities and $\gamma$-strength functions from discrete two-step $\gamma$-ray cascades in radiative proton-capture experiments
}

\author{
P. Scholz $\odot,{ }^{1, *}$ M. Guttormsen, ${ }^{2}$ F. Heim,${ }^{1}$ A. C. Larsen, ${ }^{2}$ J. Mayer, ${ }^{1}$ D. Savran $\odot,{ }^{3}$ M. Spieker,${ }^{1, \dagger}$ G. M. Tveten, ${ }^{2}$ \\ A. V. Voinov, ${ }^{4}$ J. Wilhelmy, ${ }^{1}$ F. Zeiser ${ }^{2}$ and A. Zilges ${ }^{1}$ \\ ${ }^{1}$ University of Cologne, Institute for Nuclear Physics, D-50937 Köln, Germany \\ ${ }^{2}$ Department of Physics, University of Oslo, $N-0316$ Oslo, Norway \\ ${ }^{3}$ GSI Helmholtzzentrum für Schwerionenforschung GmbH, Planckstr. 1, D-64291 Darmstadt, Germany \\ ${ }^{4}$ Department of Physics and Astronomy, Ohio University, Athens, Ohio 45701, USA
}

(Received 2 September 2019; revised manuscript received 4 February 2020; accepted 30 March 2020; published 27 April 2020)

\begin{abstract}
Background: Reaction rates of radiative capture reactions can play a crucial role in the nucleosynthesis of heavy nuclei in explosive stellar environments. These reaction rates depend strongly on $\gamma$-ray decay widths in the reaction products, which are, for nonresonant capture reactions at high excitation energies, derived from the $\gamma$-ray strength function and the nuclear level density. Recently, the ratio method was applied to primary $\gamma$ rays observed from $(d, p)$ reactions and nuclear resonance fluorescence measurements to extract the dipole strength in atomic nuclei and to test the generalized Brink-Axel hypothesis.

Purpose: The purpose of this work is to apply the ratio method to primary $\gamma$-ray intensities of the ${ }^{63,65} \mathrm{Cu}(p, \gamma)$ reactions to extract $\gamma$-ray strength information on the nuclei ${ }^{64,66} \mathrm{Zn}$. The impact of spin distribution, total $\gamma$-ray decay widths, level densities, and width fluctuations on the application of the ratio method will be discussed. Additionally, by comparing the relative $\gamma$-ray strength at different excitation energies, conclusions on the validity of the generalized Brink-Axel hypothesis can be made.

Method: The radiative proton capture reaction measurements have been performed at the HORUS $\gamma$-ray spectrometer of the University of Cologne at one excitation energy for each reaction. Primary $\gamma$-ray intensities have been determined by normalizing secondary $\gamma$-ray transitions in two-step cascades using their absolute branching ratio. The ratio method was applied to the measured primary $\gamma$-ray intensities as well as to previous measurements by Erlandsson $e t$ al. at different excitation energies.

Results: The relative strength function curve for ${ }^{64} \mathrm{Zn}$ from our measurement shows no significant deviation from the previous measurement at a different excitation energy. The same is true for ${ }^{66} \mathrm{Zn}$ where both measurements were at almost the same excitation energy. Absolute $\gamma$-strength function values have been obtained by normalizing the relative curves to quasiparticle random phase approximation calculations because of the absence of experimental data in the respective energy region.

Conclusion: The generalized Brink-Axel hypothesis, i.e., the independence of the strength function on the excitation energy, seems to hold in the studied energy region and nuclei. The method to obtain primary $\gamma$-ray intensities from two-step cascade spectra was shown to be a valuable and sensitive tool although its uncertainties are connected to the knowledge of the low-energy level scheme of the investigated nucleus. The scaling in the ratio method should be taken with care, because the relative strength is not a simple sum of $f_{E 1}$ and $f_{M 1}$ but a somewhat complex linear combination dependent on the excitation energy of the nucleus.
\end{abstract}

DOI: 10.1103/PhysRevC.101.045806

\section{INTRODUCTION}

Almost all heavy nuclei in our cosmos are produced via sequences of radiative neutron-capture reactions in either the $s$ or the $r$ process [1-3]. Recent astrophysical model investigations show that, especially, the $r$-process path may

\footnotetext{
*Present address: Department of Physics, University of Notre Dame, Indiana 46556-5670, USA; pscholz@nd.edu

${ }^{\dagger}$ Present address: Department of Physics, Florida State University, Tallahassee, FL 32306, USA.
}

depend more strongly on $(n, \gamma)$ reaction rates than previously assumed $[4,5]$. These rates can be altered dramatically by structures in the low-energy tail of the $\gamma$-ray strength function $[6,7]$. The larger the neutron excess of the isotopes, the larger the potential impact of structures like the pygmy dipole resonance [8] and the low-energy up-bend [9-11]. Despite these structures, fundamental questions about the $\gamma$ ray strength function need to be explored to reliably predict cross sections of reactions involving $\gamma$ rays: How do emission and absorption probabilities of $\gamma$ rays relate to each other? Is the $\gamma$-ray strength of nuclei independent of the excitation mechanism, excitation energy and the involved levels? 
If not, where are the limits of the generalized Brink-Axel hypothesis [12-14]? The answers to these questions require thorough experimental studies using different techniques and probes. Moreover, dependencies in the extraction of the $\gamma$-ray strength function from experimental data on other nuclear physics parameters, such as nuclear level densities or spin distributions, need to be minimized and to be under control. Throughout the last years, many techniques have been developed and improved (see [15-28] and references therein) to investigate $\gamma$-strength functions above and below the particleseparation energies. All of these approaches are to some extent selective regarding their excitation mechanism and some rely on theoretical modeling in the determination of the $\gamma$-strength function.

Surprising deviations have been found for the $\gamma$-strength function in ${ }^{90} \mathrm{Zr}$. Results of $\gamma$-ray induced measurements [17] —used as input for statistical model calculationswere not able to reproduce the partial cross sections of the ${ }^{89} \mathrm{Y}(p, \gamma)$ reactions via the statistical model [18]. Deviations between different experimental techniques were also reported for ${ }^{120} \mathrm{Sn}$ [25], while in the case of ${ }^{96} \mathrm{Mo}$ [16] various methods seem to agree. Furthermore, a new method using the $\left(\gamma, \gamma^{\prime} \gamma^{\prime \prime}\right)$ reactions has revealed inconsistencies in the extraction of $\gamma$-strength function values in ${ }^{128} \mathrm{Te}$ from different excitation energies pointing to a violation of the generalized Brink-Axel hypothesis in that specific case [21]. Hence, there is a clear need for further investigations.

Here we report on the measurement of primary $\gamma$-ray intensities for radiative proton-capture reactions on ${ }^{63,65} \mathrm{Cu}$ to extract $\gamma$-strength information on the nuclei ${ }^{64,66} \mathrm{Zn}$ by adopting the ratio method [23,26]. For the first time at the HORUS $\gamma$-ray spectrometer, these primary $\gamma$-ray intensities were derived from coincident secondary $\gamma$ rays using their absolute branching ratios. With this we are able to extend available $\gamma$-ray strength data as well as compare relative dipole strength functions to previous measurements at different excitation energies obtained by a different experimental technique. At first, however, a more detailed derivative of the ratio method formulas for the application to radiative protoncapture reactions will be given.

\section{THE DIPOLE STRENGTH FROM PRIMARY $\boldsymbol{\gamma}$-RAY TRANSITION INTENSITIES IN RADIATIVE CAPTURE REACTIONS}

Because of general dependencies on nuclear physics parameters like optical-model potentials and nuclear level densities, primary $\gamma$-ray intensities cannot be used directly to obtain $\gamma$-strength function values. In the past, so-called partial cross sections obtained for $(p, \gamma)$ reactions at many different excitation energies were compared to statistical model calculations. By fixing the nuclear level density and the opticalmodel potential to total reaction cross sections, it was argued that remaining deviations for the partial cross sections can be traced back to the $\gamma$-strength functions [18,29]. The ratio between theoretical and experimental partial cross section was used to modify the dipole strength in the reaction product. However, because various combinations of nuclear level density models and optical-model potentials might lead to an equally good description of total $(p, \gamma)$ cross sections, the theoretical uncertainties can be large.

Such uncertainties can be reduced by using the ratios of primary $\gamma$-ray intensities to various final states with the same spin and parity similar to Refs. [21,23,26,27].

If the statistical decay of excited states is independent of the excitation mechanism, the intensity $I_{L}$ of primary $\gamma$-ray transitions measured depends on the spin distribution $s\left(J^{\pi}\right)=$ $s_{j}$ in the compound nucleus, the average $\gamma$-decay width $\left\langle\Gamma_{i \rightarrow L}\right\rangle_{j}$ of the levels $i$ with spin and parity $j$ in the excitation energy bin to the final state $L$ and the width fluctuation factor,

$$
\omega_{j}=\frac{\left\langle\Gamma_{i, \text { tot }}\right\rangle_{j}}{\left\langle\Gamma_{i \rightarrow L}\right\rangle_{j}} \times\left\langle\frac{\Gamma_{i, \text { tot }}}{\Gamma_{i \rightarrow L}}\right\rangle_{j},
$$

which enables the separation of the expectation values:

$$
\begin{aligned}
I_{\gamma_{L}} & =\sum_{j}\left(s_{j} \times \frac{1}{n} \sum_{i}^{n} \frac{\Gamma_{i \rightarrow L}}{\Gamma_{i, \text { tot }}}\right) \\
& =\sum_{j}\left(s_{j} \omega_{j} \times \frac{\left\langle\Gamma_{i \rightarrow L}\right\rangle_{j}}{\left\langle\Gamma_{i, \text { tot }}\right\rangle_{j}}\right) .
\end{aligned}
$$

The inner sum runs over all levels $i$ with spin and parity $j$. The outer sum runs over all different spins and parities $j$ in the initial excitation energy bin. Focusing only on dipole transitions, Eq. (2) can be separated into two terms, one for states $j$ which decay via $E 1$ and states $j$ ' which can decay via $M 1$ transitions to the state $L$. Using the definition of the $\gamma$-strength function from Bartholomew et al. [30],

$$
f_{i \rightarrow L}\left(E_{\gamma}\right)=\frac{\left\langle\Gamma_{i \rightarrow L}\right\rangle_{j}}{E_{\gamma}^{3}} \rho_{j}\left(E_{\gamma}\right),
$$

the intensity can be expressed as

$$
I_{\gamma_{L}} \sim E_{\gamma}^{3}\left(\sum_{j} \frac{\omega_{j} s_{j}}{\rho_{j}} \frac{f_{E 1}\left(E_{\gamma}\right)}{\left\langle\Gamma_{i, \text { tot }}\right\rangle_{j}}+\sum_{j^{\prime}} \frac{\omega_{j^{\prime}} s_{j^{\prime}}}{\rho_{j^{\prime}}} \frac{f_{M 1}\left(E_{\gamma}\right)}{\left\langle\Gamma_{i, \text { tot }}\right\rangle_{j^{\prime}}}\right) .
$$

By using the ratio of primary $\gamma$-ray transitions to two different final states $L_{1}$ and $L_{2}$ with the same spin and parity and assuming that the total $\gamma$-ray decay width of states $j-1$ and $j+1$ can be related to the one of $j$ via

$$
c_{j+1}=\frac{\left\langle\Gamma_{i, \text { tot }}\right\rangle_{j}}{\left\langle\Gamma_{i, \text { tot }}\right\rangle_{j+1}},
$$

we find

$$
\frac{I_{\gamma_{L 1}}}{I_{\gamma_{L 2}}}=\frac{f_{E 1}\left(E_{\gamma_{1}}\right)+\frac{b}{a} \times f_{M 1}\left(E_{\gamma_{1}}\right)}{f_{E 1}\left(E_{\gamma_{2}}\right)+\frac{b}{a} \times f_{M 1}\left(E_{\gamma_{2}}\right)} \times \frac{E_{\gamma_{1}}^{3}}{E_{\gamma_{2}}^{3}},
$$

with the substitutions,

$$
a=\sum_{j} \frac{s_{j} \omega_{j} c_{j}}{\rho_{j}}, b=\sum_{j^{\prime}} \frac{s_{j^{\prime}} \omega_{j^{\prime}} c_{j^{\prime}}}{\rho_{j^{\prime}}} .
$$

With these expressions it is now possible to study the $E 1$ strength function curves for different excitation mechanisms. The impact of the $M 1$ strength on the results can then be estimated via the ratio $b / a$. 
Primary $\gamma$-ray intensities have been obtained for the ${ }^{63,65} \mathrm{Cu}(p, \gamma){ }^{64,66} \mathrm{Zn}$ reactions in a way similar to the twostep cascade technique [9,31-35]. This allows not only the measurement of many more primary $\gamma$ rays at one excitation energy than using singles spectra only but also a more sensitive investigation of new branching transitions in the reaction product, as explained in the following section.

\section{PRIMARY $\gamma$-RAY INTENSITIES FROM DISCRETE TWO-STEP $\gamma$-RAY CASCADES AND $\gamma \gamma$ COINCIDENCES}

\section{A. Experimental details}

The experiments were carried out at the 10-MV FNTandem accelerator using the high-efficiency $\gamma$-ray spectrometer HORUS at the Institute for Nuclear Physics of the University of Cologne, Germany [36]. This setup consists of 14 highpurity germanium (HPGe) detectors placed at angles of $35^{\circ}$, $45^{\circ}, 90^{\circ}, 135^{\circ}$ and $145^{\circ}$ with respect to the beam axis. Six of the detectors were equipped with bismuth-germanate (BGO) shields for an active Compton background suppression.

The efficiency of the setup for low-energy $\gamma$-ray transitions was calibrated using ${ }^{226} \mathrm{Ra}$ and ${ }^{56} \mathrm{Co}$ sources. For highenergetic $\gamma$ rays a measurement of the ${ }^{27} \mathrm{Al}(p, \gamma)$ resonance at $E_{p}=3674 \mathrm{keV}$ was performed [37]. Additionally, the efficiency was simulated up to $\gamma$-ray energies of $12 \mathrm{MeV}$ using the implementation of HORUS in GEANT4 [38].

Two highly enriched targets have been used for the measurements. The isotopic enrichments were $99.73 \%$ and $99.2 \%$ for ${ }^{63} \mathrm{Cu}$ and ${ }^{65} \mathrm{Cu}$, respectively. With proton energies of $3.5 \mathrm{MeV}$ and $2.0 \mathrm{MeV}$, the beam energies were below the respective ${ }^{63,65} \mathrm{Cu}(p, n)$ thresholds. Both targets were irradiated for about 3 days each with proton beam currents of $\sim 1.0 \mu \mathrm{A}$. Via Rutherford backscattering spectrometry (RBS) measurements at the RUBION facility, Bochum, Germany, after the experiment, the thicknesses of the targets were found to be $1.55 \mathrm{mg} \mathrm{cm}^{-2}$ and $2.67 \mathrm{mg} \mathrm{cm}^{-2}$ resulting in energy losses of $87 \mathrm{keV}$ and $223 \mathrm{keV}$ for ${ }^{63} \mathrm{Cu}$ and ${ }^{65} \mathrm{Cu}$, respectively. Please note that these energy losses determine the width of the excitation energy window in the compound nucleus. In the following, the energy of the primary $\gamma$-ray transitions is obtained from

$$
E_{\gamma}=E_{\mathrm{c} . \mathrm{m} .}+Q_{p, \gamma}-\frac{\Delta E}{2}-E_{L}
$$

where $E_{\text {c.m. }}$ is the center-of-mass energy, $Q_{p, \gamma}$ the $Q$ value of the respective reaction, $\Delta E$ the energy loss in the target material, and $E_{L}$ the energy of the populated state in the reaction product.

Taking advantage of the in-beam technique with HPGe detectors, the total number of radiative proton captures $N_{(p, \gamma)}$ are determined via the yields of ground-state transitions in the reaction product (see, e.g., Refs. [36,39] and references therein). Here, contributions of 24 (21) tabulated ground-state transitions in ${ }^{64} \mathrm{Zn}\left({ }^{66} \mathrm{Zn}\right)$ have been measured, not including the direct deexcitation to the ground state. The total number of radiative proton captures $N_{(p, \gamma)}$ is used in the following to normalize the intensities of primary $\gamma$-ray transitions.
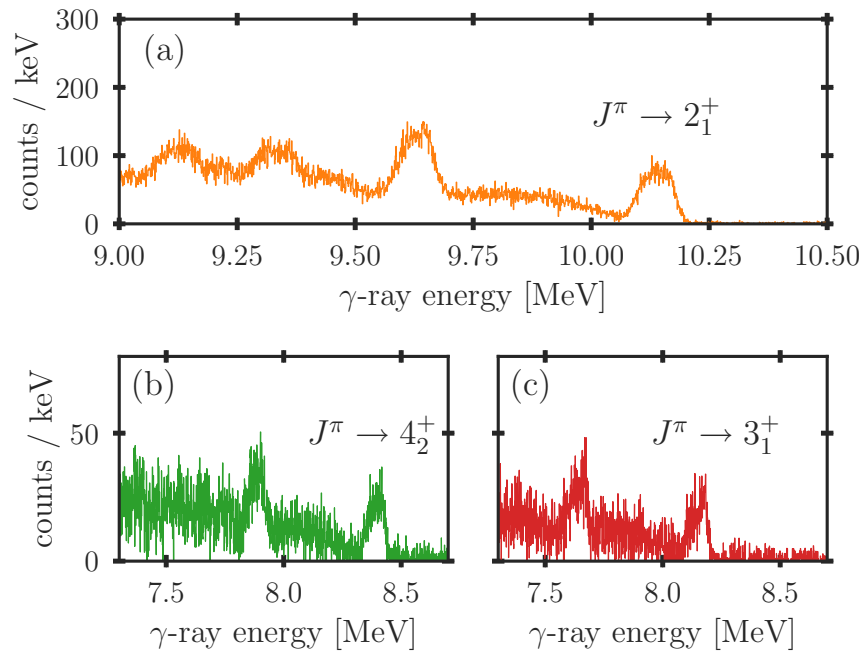

FIG. 1. High-energy coincidence spectra revealing primary $\gamma$ ray transitions in the ${ }^{63} \mathrm{Cu}(p, \gamma \gamma)$ reaction after applying gates on the secondary transitions depopulating the (a) $2_{1}^{+}$, (b) $2_{2}^{+}$, and (c) $3_{1}^{+}$ states in ${ }^{64} \mathrm{Zn}$.

\section{B. $\gamma \gamma$ coincidences}

The number of high-energetic primary $\gamma$-ray transitions which can be identified in singles spectra of HPGe detectors is limited by the high-energetic background mainly stemming from single- and double-escape peaks from higher-energetic transitions. For instance, in the cases of ${ }^{64,66} \mathrm{Zn}$ the energy of the primary $\gamma$-ray transition to the $2_{1}^{+}$state is almost equal to the energy of the single-escape peak of the primary transition to the ground state. Moreover, for the population of higher-lying states, the primary $\gamma$-ray transitions become more difficult to distinguish from each other.

The way to increase the selectivity and sensitivity of such measurements is the application of $\gamma \gamma$-coincidence techniques. For this, coincident $\gamma$-ray transitions in a time window of $500 \mathrm{~ns}$ have been sorted into a $\gamma \gamma$-coincidence matrix, from which time-correlated background events were subtracted.

Via gates on high-intense secondary transitions, primary $\gamma$-ray transitions can be revealed in the high-energy part of the coincidence spectrum. This is illustrated in Fig. 1. The spectra in Figs. 1(b) and 1(c) were obtained via gates on the $4_{2}^{+} \rightarrow$ $2_{2}^{+}$transition with $937 \mathrm{keV}$ and the $3_{1}^{+} \rightarrow 2_{2}^{+}$transition with $1180 \mathrm{keV}$, respectively. In this way, both primary transitions can be easily resolved, which would hardly be possible solely from singles spectra. The yield of the primary transition in the coincidence spectrum $N_{J^{\pi} \rightarrow L}^{\text {coinc }}$ is connected to the intensity of the primary transition $I_{\gamma_{L}}$ via

$$
I_{\gamma_{L}}=\frac{1}{N_{(p, \gamma)}} \times \frac{N_{L \rightarrow f}}{\epsilon_{g g} b_{L \rightarrow f}},
$$

where $b_{L \rightarrow f}$ is the absolute branching of the secondary $\gamma$-ray transition used for the coincidence gate.

For the ${ }^{63} \mathrm{Cu}(p, \gamma \gamma)$ reaction intensities of 42 primary $\gamma$ rays were obtained via this approach. Because the energy loss and, thus, the widths of the peaks belonging to primary $\gamma$-ray transitions were too large for the ${ }^{65} \mathrm{Cu}(p, \gamma \gamma)$ reaction, 
primary $\gamma$-ray intensities were not obtained from $\gamma \gamma$ coincidences in this case.

\section{Discrete two-step $\gamma$-ray cascades}

For the first time at the HORUS $\gamma$-ray spectrometer, the yield of primary $\gamma$ rays have been obtained by a method based on the two-step $\gamma$-ray cascade (TSC) technique [31-33]. This method was originally developed to study $\gamma$-ray strength functions and nuclear level density models in radiative neutroncapture reactions [24]. Later it was adopted for $(p, \gamma)$ reactions, which reduced the impact of width fluctuations on the obtained $\gamma$-ray spectra because of the energy loss of the protons and, hence, a wider excitation window [34,35]. Statistical $\gamma$-decay codes have been developed to simulate the spectra of $\gamma$ rays which are part of a two-step $\gamma$-ray cascade populating states in the reaction product $[40,41]$. Information on the statistical properties of the nucleus can be obtained by the reproduction of the measured spectra using these simulations; see, e.g., Refs. [9,24].

In contrast to the standard TSC method, our aim here is not to test $\gamma$-ray strength function and nuclear level density models via such simulations, but use the yield of discrete secondary $\gamma$-ray transitions to determine the intensity of primary $\gamma$ rays, which will be explained in the following.

Figure 2 illustrates the application of the TSC method for the ${ }^{63} \mathrm{Cu}(p, \gamma \gamma){ }^{64} \mathrm{Zn}$ reaction. Coincident $\gamma$-ray events within a time window of 500ns were sorted into the TSC matrix after the subtraction of the time-correlated background. In each panel in Fig. 2, the projection of the TSC matrix on the axis of the sum-peak energy of two coincident $\gamma$ rays is shown. In the high-energy part of this spectrum, peaks become visible at energies representing the population of states in ${ }^{64} \mathrm{Zn}$ and their single and double escape equivalents. Via gates on the population of the ground state and the first two $2^{+}$states one obtains the so-called TSC spectra shown in Fig. 2. These spectra were normalized to the efficiency and to the total number of radiative proton captures.

By definition, for every low-energetic $\gamma$-ray transition from an intermediate excited state $L$ to a final state $f$ in ${ }^{64} \mathrm{Zn}$ visible in those TSC spectra, there must have been a primary $\gamma$-ray transition $\gamma_{L}$ from the initial state $i$ populating this level $L$. Therefore, the intensity of the low-energy $\gamma$-ray transitions can be used to deduce the intensity of the primary transition $I_{\gamma_{L}}$ via its absolute branching $b_{L \rightarrow f}$ :

$$
I_{\gamma_{L}}=\frac{N_{i \rightarrow L}}{N_{(p, \gamma)}}=\frac{1}{N_{(p, \gamma)}} \times \frac{N_{L \rightarrow f}}{b_{L \rightarrow f} \epsilon_{t s c}} .
$$

Although this approach relies on the certainty of absolute branching ratios, it has two advantages over the usage of the high-energy primary $\gamma$-ray lines: The secondary $\gamma$-ray lines do not suffer from the energy loss of the protons in the spectrum and, hence, allow a more precise selection of the populated (intermediate) states. Furthermore, the statistics can be determined much more reliably because the peaks are less smeared out than those of the primary transitions.

Figure 3 shows the low-energy part of the TSC spectra for the ${ }^{63} \mathrm{Cu}(p, \gamma \gamma)$ measurement together with the adopted level schemes of ${ }^{64} \mathrm{Zn}$. The single TSC spectra are drawn
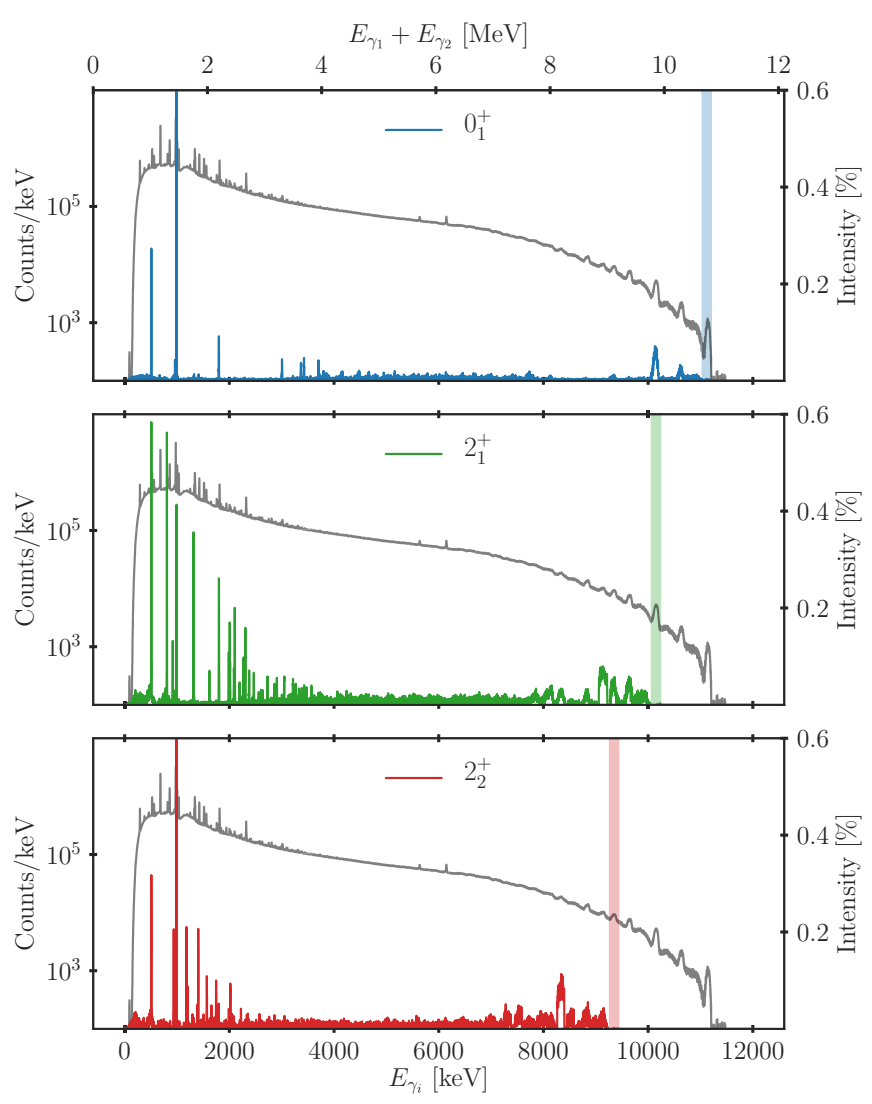

FIG. 2. The projection of the TSC matrix on the axis of summed energies for ${ }^{64} \mathrm{Zn}$ (gray) and the TSC spectra for gates on cascade energies for the population of the ground state (top), the $2_{1}^{+}$(middle), and the $2_{2}^{+}$state (bottom). The binning for these spectra is $1 \mathrm{keV}$. By default, the number of events is symmetric around half of the cascade energy. However, discrete $\gamma$-ray lines in the low-energy part of the spectra are easier to distinguish from each other than those in the high-energy region because of their smaller width.

with an offset of the level energy corresponding to the final states in the two-step cascade selected. With this, transitions feeding the selected final state can directly be assigned to the corresponding intermediate level L.

Because of the gate on the sum spectra and the subtraction of energy-correlated background events, the only background visible in these spectra are false two-step cascades yielding the same sum energy: (i) $511 \mathrm{keV}+$ a single-escape event of the direct population of the final state; (ii) both parts of a Compton scattered $\gamma$ ray directly populating the final state; (iii) a double-escape event + the energy of the $2_{1}^{+} \rightarrow 0_{1}^{+}$ transition. The latter one is specific for these reactions because the level energies of the $2_{1}^{+}$states in ${ }^{64,66} \mathrm{Zn}$ are at $991 \mathrm{keV}$ and $1039 \mathrm{keV}$ and, thus, not distinguishable from $1022 \mathrm{keV}$ within the width of the gate on the sum-peak spectra. In total 72 (75) discrete $\gamma$-ray transitions were analyzed for the TSC spectra of ${ }^{64} \mathrm{Zn}\left({ }^{66} \mathrm{Zn}\right)$ shown in Fig. 3.

\section{Results on primary $\gamma$-ray intensities}

Primary $\gamma$-ray intensities have been calculated according to Eqs. (9) and (10) using known branching ratios from 


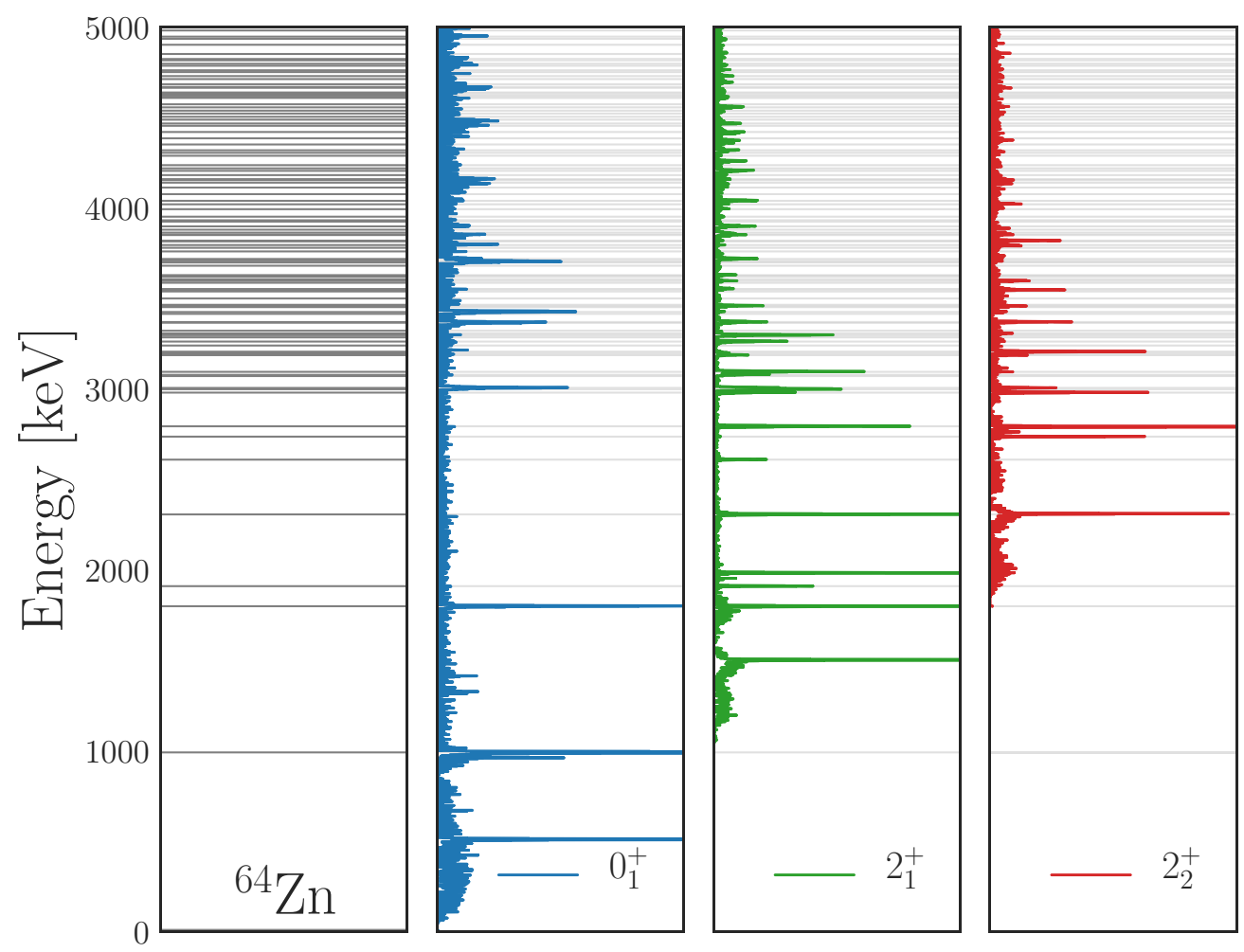

FIG. 3. Level scheme (gray solid and dashed lines) and TSC spectra for ${ }^{64} \mathrm{Zn}$. The TSC spectra shown were obtained via gates in the sum-peak spectrum on the energies of the population of the three lowest lying states. For the two excited states, the TSC spectra are plotted with an offset corresponding to the respective level energy of the populated states. In this way, transitions from an excited state become directly visible. Levels with no respective transitions in the TSC spectra are either not populated or not branching to these three final states.

Ref. [42]. Of all analyzed transitions in the discrete TSC spectra, as well as in the $\gamma \gamma$-coincidence spectra, some belong to the population of the same excited states. In these cases, weighted averages have been used to determine the resulting intensity. In total, primary intensities for the population of 37 (49) unique excited states in ${ }^{64} \mathrm{Zn}\left({ }^{66} \mathrm{Zn}\right)$, which are shown in Fig. 4, have been measured. Tables I and II list relative intensities normalized on the highest-energetic transitions to simplify the comparison to the results of Ref. [43].

Figure 4 also shows the results of Ref. [43]. These were measured via the average resonance method, which was first introduced for neutron capture reactions [44-46]. In their work, they used a $175 \mu \mathrm{g} \mathrm{cm}^{-2}$ thick ${ }^{63} \mathrm{Cu}$ and a $200 \mu \mathrm{g} \mathrm{cm}^{-2}$ thick ${ }^{65} \mathrm{Cu}$ target. Proton energies were averaged between $E_{p}=2.1-3.1 \mathrm{MeV}$ and $E_{p}=1.7-2.2 \mathrm{MeV}$ for ${ }^{63} \mathrm{Cu}$ and ${ }^{65} \mathrm{Cu}$, respectively. This results in averaged excitation energies of $E_{x}=10.31 \mathrm{MeV}$ for ${ }^{64} \mathrm{Zn}$ and $E_{x}=10.82 \mathrm{MeV}$ for ${ }^{66} \mathrm{Zn}$, i.e., $\approx 800 \mathrm{keV}$ lower for ${ }^{64} \mathrm{Zn}$ and almost the same for ${ }^{66} \mathrm{Zn}$ compared to our measurement.

\section{RATIOS OF PRIMARY $\gamma$ RAYS TO FINAL STATES WITH $J^{P}=0^{+}, 1^{+} 2^{+}, 4^{+}$}

\section{A. Impact of $M 1$ strength}

Equation (6) can be used to construct a relative dipolestrength curve for ${ }^{64,66} \mathrm{Zn}$ from these primary $\gamma$-ray intensities if the coefficients $a, b$ in Eq. (6) can be approximated, i.e., the impact of the spin distribution $s_{j}$, the level density per spin and parity $\rho_{j}$, the deviation in total $\gamma$ decay width $c_{j}$, and the width-fluctuation factors $\omega_{j}$. For both reactions, the spin distribution factors $s_{j}$ have been calculated from the compound formation cross sections using the TALYS code $[47,48]$ and the semimicroscopical JLM proton+nucleus optical-model potential [49]. For different normalizations of this potential, implemented in TALYS and as described in Ref. [50], no significant differences in the results have been observed. The population is dominated by negative-parity states with a $\sim 20 \%$ contribution of positive ones. For most phenomenological level-density models as the widely used Gilbert-Cameron model [51], the level density is independent of the parity of interest, i.e., $\rho_{j}=\rho_{j \prime}$. Moreover, at excitation energies above $10 \mathrm{MeV}$, no significant differences were found for the level density of opposite parities from microscopical approaches as in [52]. Experimentally, no differences for the level density of $J=2$ states with positive or negative parity in ${ }^{58} \mathrm{Ni}$ and ${ }^{90} \mathrm{Zr}$ were found [53]. Several methods can be used to compute the width fluctuation factors within TALYS: the Hofmann-Richert-Tepel-Weidenmüller model (HRTW) [54], Moldauer model [55], and the Gaussian orthogonal ensemble (GOE) of Hamiltonian matrices [56]. All methods have been compared in Ref. [57] for total elastic as well as radiative capture reactions. For the latter ones it was found that above $1 \mathrm{MeV}$ incident beam energy, i.e., if there are enough open decay channels, the impact of width fluctuation corrections vanishes $(\omega \approx 1)$. 
TABLE I. Relative intensities of primary $\gamma$ rays from this work and Ref. [43] for ${ }^{64} \mathrm{Zn}$. $\gamma$-ray energies from this work are obtained from the excitation energy minus half of the energy loss. Intensities without given uncertainty are lower limits because of uncertain absolute branching. New level energies are marked with an asterisk.

\begin{tabular}{|c|c|c|c|c|c|}
\hline$E_{L}(\mathrm{MeV})$ & $J^{\pi}$ & $\begin{array}{c}E_{\gamma}(\mathrm{MeV}) \\
\text { Ref. [43] }\end{array}$ & $\begin{array}{c}I_{\text {rel }} \\
\text { Ref. [43] }\end{array}$ & $\begin{array}{c}E_{\gamma}(\mathrm{MeV}) \\
\text { This work }\end{array}$ & $\begin{array}{c}I_{\text {rel }} \\
\text { This work }\end{array}$ \\
\hline 0.000 & $0+$ & 10.31 & $1.000(125)$ & 11.12 & $1.000(057)$ \\
\hline 0.992 & $2+$ & 9.32 & $2.203(272)$ & 10.13 & $2.088(079)$ \\
\hline 1.799 & $2+$ & 8.51 & $1.308(163)$ & 9.32 & $1.200(184)$ \\
\hline 1.910 & $0+$ & 8.40 & $0.242(038)$ & 9.21 & $0.559(075)$ \\
\hline 2.307 & $4+$ & 8.00 & $0.562(073)$ & 8.81 & $0.772(031)$ \\
\hline 2.609 & $0+$ & 7.70 & $0.152(025)$ & 8.51 & $0.135(026)$ \\
\hline 2.737 & $4+$ & 7.57 & $0.430(058)$ & 8.38 & $0.414(031)$ \\
\hline 2.793 & $2+$ & 7.52 & $0.542(072)$ & 8.33 & $0.774(013)$ \\
\hline 2.980 & $3+$ & 7.33 & $0.540(077)$ & 8.14 & $0.757(053)$ \\
\hline 2.998 & $3-$ & - & - & 8.12 & $0.732(328)$ \\
\hline 3.006 & $2+$ & - & - & 8.11 & $0.534(066)$ \\
\hline 3.079 & $4+$ & 7.23 & $0.302(077)$ & 8.04 & $0.415(157)$ \\
\hline 3.095 & $(3)+$ & 7.22 & $0.476(081)$ & 8.02 & $0.523(215)$ \\
\hline 3.187 & $1+$ & - & - & 7.93 & $0.284(043)$ \\
\hline 3.197 & $(2,3)$ & - & - & 7.92 & $0.074(007)$ \\
\hline 3.206 & $(3)+$ & - & - & 7.91 & $0.667(142)$ \\
\hline 3.262 & 1 & - & - & 7.86 & $0.317(050)$ \\
\hline 3.297 & $(2)+$ & - & - & 7.82 & $0.782(065)$ \\
\hline 3.366 & $1+$ & - & - & 7.75 & $0.339(057)$ \\
\hline 3.370 & $3+$ & - & - & 7.75 & $0.610(222)$ \\
\hline 3.425 & $1+$ & 6.89 & $0.273(042)$ & 7.69 & $0.180(050)$ \\
\hline $3.454(1)^{*}$ & - & - & - & 7.67 & 0.037 \\
\hline 3.459 & $(2,3)$ & - & - & 7.66 & $0.281(077)$ \\
\hline 3.546 & $(\leqslant 3)$ & - & - & 7.57 & $0.164(127)$ \\
\hline 3.552 & $4+$ & - & - & 7.57 & $0.150(104)$ \\
\hline 3.597 & $(2,3,4)$ & 6.71 & $0.247(037)$ & 7.52 & $0.147(099)$ \\
\hline 3.628 & $(4+)$ & 6.68 & $0.051(019)$ & 7.49 & $0.067(028)$ \\
\hline $3.707(1)^{*}$ & $(2+)$ & - & - & 7.42 & $0.199(008)$ \\
\hline 3.718 & $(0+: 4+)$ & - & - & 7.40 & 0.139 \\
\hline 3.795 & $1+$ & 6.51 & $0.209(042)$ & 7.32 & $0.126(050)$ \\
\hline 3.819 & $(0+: 4+)$ & 6.49 & $0.269(035)$ & 7.30 & $0.234(044)$ \\
\hline 3.851 & $(\leqslant 3)+$ & - & - & 7.27 & $0.182(172)$ \\
\hline 3.853 & $5+$ & - & - & 7.27 & 0.052 \\
\hline 3.899 & $(2+, 3,4+)$ & 6.41 & $0.189(031)$ & 7.22 & $0.221(020)$ \\
\hline 3.925 & $5-$ & 6.39 & $0.075(023)$ & - & - \\
\hline 3.952 & $(3+, 4+)$ & - & - & 7.17 & $0.027(003)$ \\
\hline 4.020 & $(2+, 3+)$ & - & - & 7.10 & $0.081(078)$ \\
\hline 4.039 & $(0+: 4+)$ & - & - & 7.08 & $0.235(047)$ \\
\hline 4.077 & $(5)+$ & - & - & 7.01 & $0.032(007)$ \\
\hline $4.115(1)^{*}$ & $2+$ & - & - & 7.01 & 0.023 \\
\hline $4.135(1)^{*}$ & $(1+, 2+)$ & - & - & 6.98 & 0.049 \\
\hline 4.157 & $5-$ & - & - & 6.96 & $0.119(091)$ \\
\hline 4.205 & $(3+, 4+)$ & 6.11 & $0.152(026)$ & 6.91 & $0.207(007)$ \\
\hline $4.258(1)^{*}$ & - & - & - & 6.86 & 0.139 \\
\hline $4.311(1)^{*}$ & - & - & - & 6.81 & 0.039 \\
\hline 4.319 & $(3+, 4+)$ & - & - & 6.80 & $0.070(004)$ \\
\hline $4.357(1)^{*}$ & $(1+, 2+, 3+)$ & - & & 6.76 & 0.052 \\
\hline $4.374(1)^{*}$ & - & - & - & 6.75 & 0.110 \\
\hline $4.377(1)^{*}$ & - & - & - & 6.74 & 0.054 \\
\hline $4.415(1)^{*}$ & - & - & - & 6.70 & 0.070 \\
\hline 4.467 & $(0+)$ & - & - & 6.65 & 0.111 \\
\hline $4.559(1)^{*}$ & - & - & - & 6.56 & 0.127 \\
\hline
\end{tabular}

TABLE II. Relative intensities of primary $\gamma$ rays from this work and Ref. [43] for ${ }^{66} \mathrm{Zn}$. $\gamma$-ray energies from this work are obtained from the excitation energy minus half of the energy loss. Intensities without given uncertainty are lower limits because of uncertain absolute branching. New level energies are marked with an asterisk.

$$
E_{\gamma}(\mathrm{MeV}) \quad I_{\text {rel }} \quad E_{\gamma}(\mathrm{MeV}) \quad I_{\text {rel }}
$$

$E_{L}(\mathrm{MeV}) \quad J^{\pi} \quad$ Ref. [43] Ref. [43] This work This work

\begin{tabular}{rrrrrr}
\hline 0.000 & $0+$ & 10.82 & $1.000(128)$ & 10.72 & $1.000(150)$ \\
1.039 & $2+$ & 9.78 & $1.779(222)$ & 9.68 & $2.380(036)$
\end{tabular}

$\begin{array}{llllll}1.872 & 2+ & 8.95 & 0.995(126) & 8.85 & 1.177(019)\end{array}$

$\begin{array}{llllll}2.372 & 0+ & 8.45 & 0.180(025) & 8.35 & 0.226(005)\end{array}$

$\begin{array}{llllll}2.450 & 4+ & 8.37 & 0.238(032) & 8.27 & 0.217(003)\end{array}$

$\begin{array}{llllll}2.704 & (3) & 8.12 & 0.280(036) & 8.02 & 0.633(477)\end{array}$

$\begin{array}{llllll}2.766 & 4+ & 8.05 & 0.162(073) & 7.96 & 0.137(005)\end{array}$

$\begin{array}{llllll}2.780 & 2+ & 8.04 & 0.511(082) & 7.94 & 0.515(015)\end{array}$

$\begin{array}{llllll}2.827 & 3- & 7.99 & 0.222(034) & 7.90 & 0.226(005)\end{array}$

$\begin{array}{llllll}2.938 & 2+ & 7.88 & 0.539(071) & 7.78 & 0.928(017)\end{array}$

3.078

3.104

3.212

3.229

3.331

3.381

3.427

3.432

3.507

3.532

3.576

3.670

3.689

3.738

3.753

3.773(1)*

3.791

3.806

3.825

3.882

3.924

3.946

4.018

4.086

4.119

4.295

4.321

4.327

$4.426(1)^{*}$

$\begin{array}{lll}2+ & 7.88 & 0.539(071) \\ + & 7.74 & 0.135(021)\end{array}$

-

$0+\quad 7.72 \quad 0.141(021)$

$2+\quad 7.61 \quad 0.489(098)$

$1+\quad 7.59 \quad 0.518(085)$

$\begin{array}{ll}7.61 & 0.097(003)\end{array}$

$7.51 \quad 1.109(102)$

$7.490 .580(022)$

$\begin{array}{lllll}2+ & 7.49 & 0.338(052) & 7.39 & 0.559(120)\end{array}$

$\begin{array}{lllll}1- & 7.44 & 0.379(052) & 7.34 & 0.312(149)\end{array}$

$\begin{array}{llllll}(1,2-) & - & & - & 7.30 & 0.047(002)\end{array}$

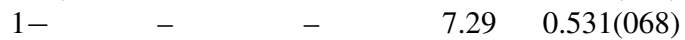

$\begin{array}{llllll}2+ & - & - & - & 7.22 & 0.215(007)\end{array}$

$\begin{array}{lllll}0+ & 7.29 & 0.094(023) & 7.19 & 0.080(003)\end{array}$

$\begin{array}{lllll}4+ & 7.24 & 0.140(020) & 7.15 & 0.219(008)\end{array}$

$\begin{array}{lllll}2+ & 7.15 & 0.299(094) & 7.05 & 0.879(020)\end{array}$

$\begin{array}{lllll}(1,2,3+) & 7.13 & 0.108(042) & 7.04 & 0.088(003)\end{array}$

$\begin{array}{llllll}(+) & - & & - & 6.99 & 0.152(009)\end{array}$

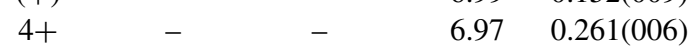

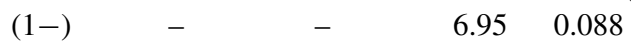

$\begin{array}{llllll}1+ & - & & - & 6.93 & 0.489(035)\end{array}$

$\begin{array}{ll}6.91 & 0.079\end{array}$

$\begin{array}{ccccc}0+ & - & - & 6.90 & 0.041(002) \\ (2)+ & - & - & 6.84 & 0.057(005)\end{array}$

$6.80 \quad 0.152(009)$

$\begin{array}{ccccc}(1-) & - & - & 6.78 & 0.116 \\ 2+ & 6.80 & 0.192(027) & 6.71 & 0.076\end{array}$

$1+\quad 6.73 \quad 0.260(034)$

$6.640 .354(009)$

6.610 .106

$(1-)$

$1+$

$-$

- $189(043)$

$6.43 \quad 0.217(008)$

$6.40 \quad 0.132(004)$

4.454

$2+$

(1)

4.461

$4.515(1)^{*}(0+/ 2+)$

4.531(1)*

4.612(1)*

4.653(1)*

$4.736(1)^{*}$

4.747(1)*

4.806

4.849

$\begin{array}{cc}- & - \\ 6.49 & 0.171(048)\end{array}$

$\begin{array}{ll}6.30 & 0.069\end{array}$

$6.27 \quad 0.147$

$6.26 \quad 0.225(007)$

$6.21 \quad 0.028$

$6.20 \quad 0.326$

$6.11 \quad 0.109$

$6.07 \quad 0.046$

$5.99 \quad 0.056$

$5.98 \quad 0.099$

$5.92 \quad 0.115(005)$

$5.87 \quad 0.261(014)$

$5.81 \quad 0.157$ 

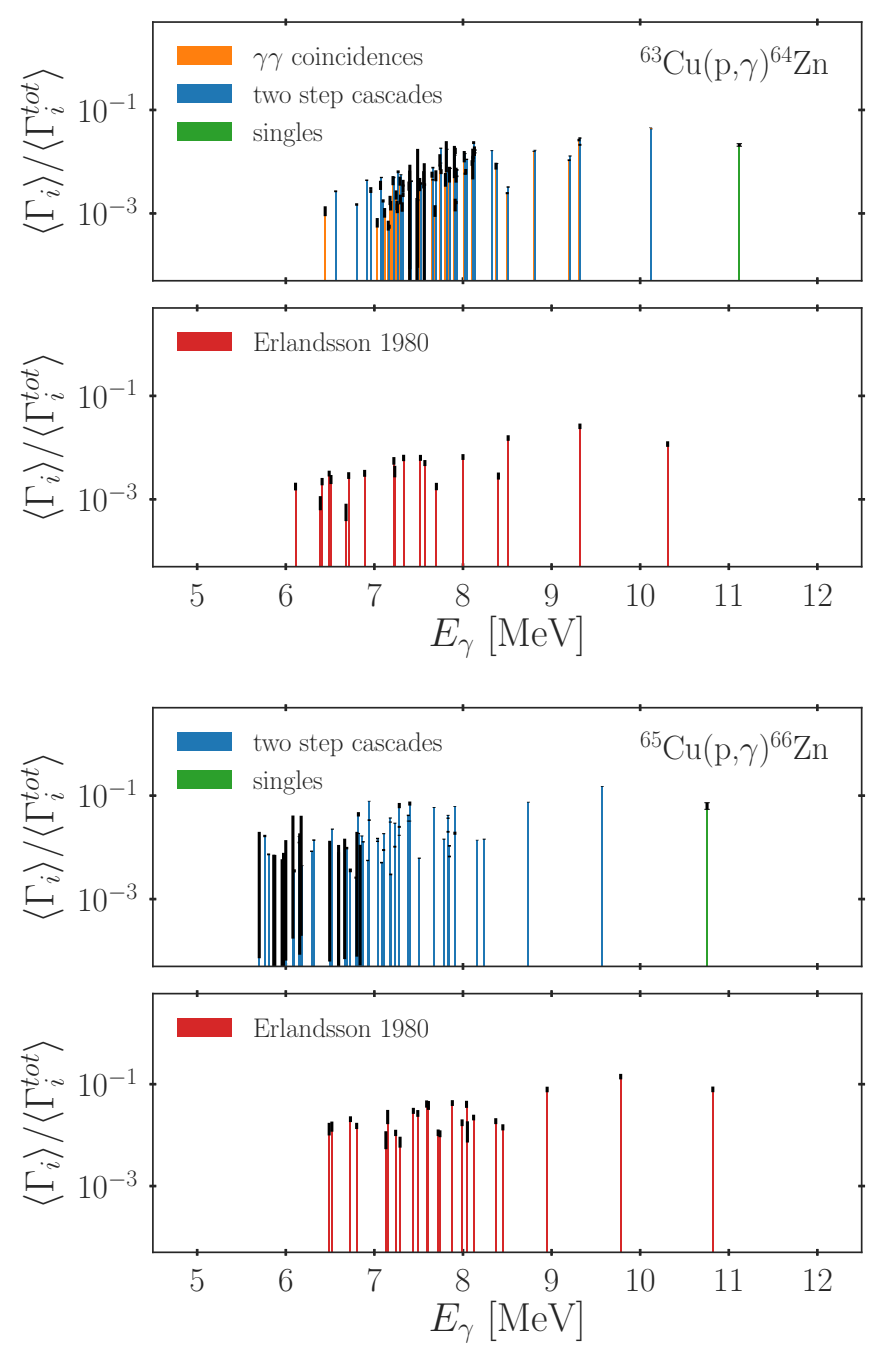

FIG. 4. Primary $\gamma$-ray intensities obtained in this work compared to the ones of Ref. [43]. Data obtained from normal $\gamma \gamma$ coincidences have been plotted with a small offset of $\Delta E_{\gamma}=10 \mathrm{keV}$ to distinguish them from the TSC results.

Hence, relative strength values have been calculated for final states with four different spins and parities: $0^{+}, 1^{+}$, $2^{+}$, and $4^{+}$. Ratios $b / a$ have been calculated based on the parameters for the Gilbert-Cameron model experimentally constrained in [58]. The ratio for final $2^{+}$states are $(b / a)_{2^{+}}=$ 0.184 and $(b / a)_{2^{+}}=0.187$ for ${ }^{64} \mathrm{Zn}$ and ${ }^{66} \mathrm{Zn}$, respectively. From TALYS calculations we see that the relative population for different spins in the excitation energy window does not change significantly for our energies and those of Ref. [43] and, thus, the ratios $(b / a)$ are the same for both measurements. Hence, if the Brink-Axel hypothesis is valid, the relative strength-function curves for both excitation energies should be the same.

\section{B. Scaling of curves for different final spins and parities}

The relative intensities of the primary $\gamma$-ray transitions of Tables I and II have been used to calculate strength-function ratios for each individual measurement and final spins and parities via

$$
f\left(E_{\gamma 2}\right)=f\left(E_{\gamma 1}\right)\left(\frac{E_{\gamma 1}}{E_{\gamma 2}}\right)^{3} \frac{I_{\gamma_{2}}}{I_{\gamma_{1}}} .
$$

In older studies such as Ref. [43], scaling factors for relative strength function curves for different final spins and parities were obtained from Hauser-Feshbach expressions, which rely on estimates for the nuclear level density and spin distribution in the specific nucleus. On the contrary, recent applications of this ratio method $[21,23,26,27]$ get rid of this model dependence by exploiting the assumption that the $\gamma$-ray strength function for a specific multipole transition has to be independent of the involved spins and parities and only depends on the energy of the $\gamma$-ray transition. The same approach is used here.

Thus, the curves obtained from the values of Ref. [43] have been scaled to our data via averaged scaling factors in two steps. First, the curves for specific $J^{\pi}$ for both measurements have been scaled to each other and, second, all curves were normalized to the $2^{+}$curve. Hereby, scaling factors were calculated from the weighted mean of interpolated strength values for the same $\gamma$-ray energies as Ref. [43].

The exceptions are the values for the population of $1^{+}$ states because of the small overlap with the $2^{+}$curves. In the case of ${ }^{66} \mathrm{Zn}, 1^{+}$data points have been scaled using a Gaussian moving average for all other points to minimize the systematic uncertainty of the scaling. For values from Ref. [43] corresponding to the $1^{+}$states in ${ }^{66} \mathrm{Zn}$, the $2^{+}$curve was extrapolated by an exponential function to lower $\gamma$-ray energies.

The resulting curves are shown in Fig. 5. In the case of ${ }^{64} \mathrm{Zn}$ (see top panels in Fig. 5), the new data allow us to compare the $\gamma$-ray strength for transitions to different final spins and parities for two different excitation energies. This comparison shows that the trend in strength for both experiments is similar, which is to be expected if the $\gamma$-ray strength observed is independent of the excitation energy. Therefore, in the studied excitation energy range for this nucleus, the generalized Brink-Axel hypothesis can be assumed to be valid.

In the case of ${ }^{66} \mathrm{Zn}$, the excitation energies are basically the same. Thus, even without assuming the generalized BrinkAxel hypothesis to be valid, we expect the $\gamma$-ray strength to be the same for both experiments. The curves shown in the bottom panels of Fig. 5 support this. Looking at the results for $J_{f}^{\pi}=2^{+}$and $J_{f}^{\pi}=4^{+}$, an increase in the $\gamma$-ray strength at around $7.0 \mathrm{MeV}$ is visible. For $J_{f}^{\pi}=2^{+}$points based on Ref. [43], this enhancement is not as strong as for our data points. This might be because of the average width of the excitation energy window in the compound nucleus. In Ref. [43], the data was obtained by averaging over a proton energy range between $1.7 \mathrm{MeV}$ and $2.2 \mathrm{MeV}$, resulting in a width of the energy window of about $500 \mathrm{keV}$. As reported above, the target thickness in this experiment was $223 \mathrm{KeV}$, i.e., roughly half as wide. 

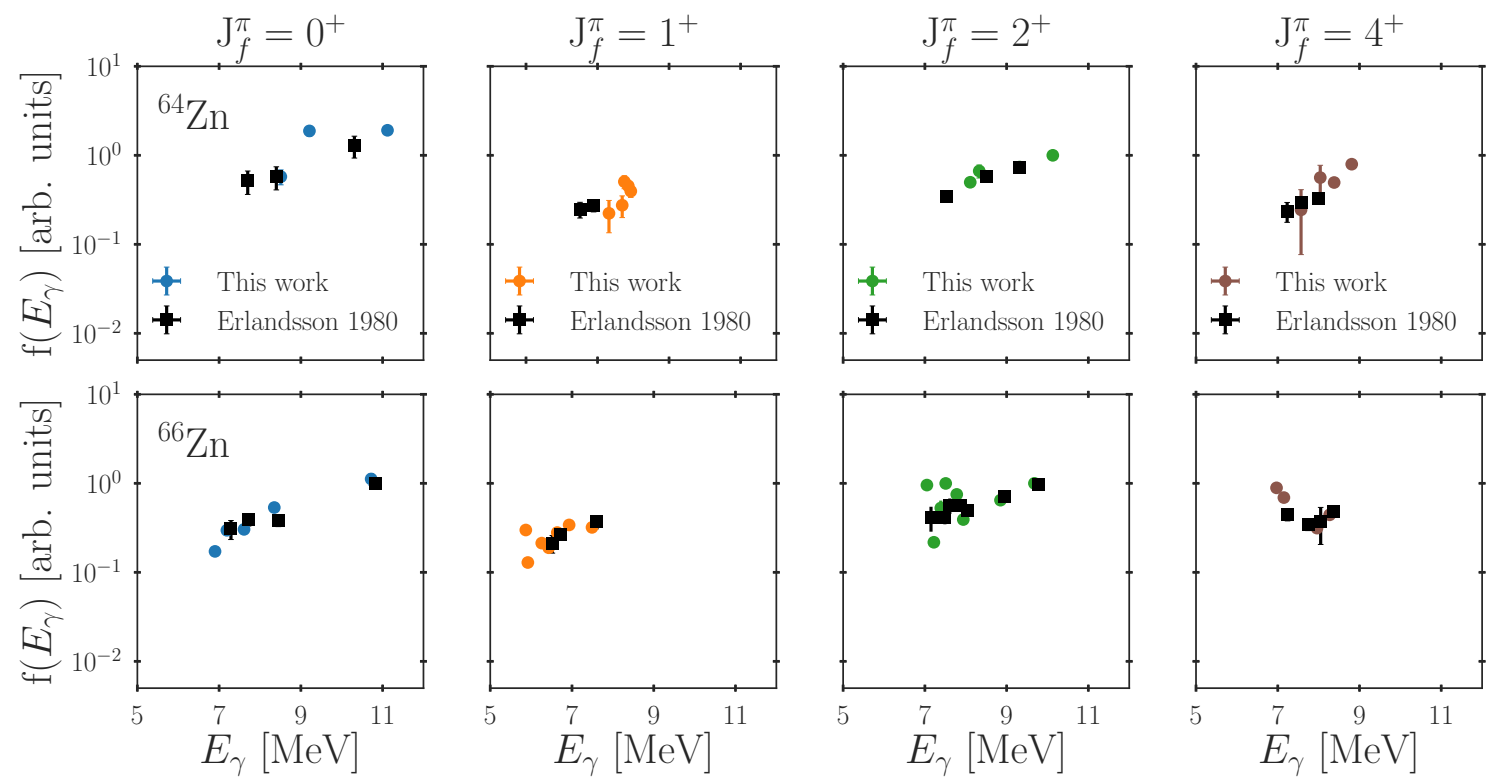

FIG. 5. Ratios of $\gamma$-strength values obtained from experimental data for both zinc isotopes for final states with same spin and parity. The ratios for the primary $\gamma$-ray intensities of this work (full circles) and the ones from Ref. [43] (full squares) have been calculated separately and were normalized towards each other.

\section{THE DIPOLE STRENGTH FUNCTION IN ${ }^{64,66} \mathrm{Zn}$}

Because the relative $\gamma$-ray strength should be independent of the spin and parity of the final states [12,13], all curves of Fig. 5 can be merged to one.

For a comparison to other experimental techniques, the relative values have to be normalized to absolute ones. Because of the absence of other experimental data in this energy region, the values were normalized with respect to the microscopical strength function of the Gogny D1M HFB+QRPA calculations $[59,60]$ at the transition energy for the population of the $2_{1}^{+}$in ${ }^{64,66} \mathrm{Zn}$ with the newest modifications suggested by Ref. [61]. In Sec. IV A, we found that the strength we measured is not purely $E 1$ but still has a contribution of about $\approx 18 \% M 1$, which was included for the absolute normalization. The resulting relative strength values are listed in Table III for both nuclei.

Figure 6 shows the comparison of our results to the experimental results of $\gamma$-ray induced experiments above the particle thresholds. Additionally, bands for a Gaussian moving average have been added for values close in energy to make the general trend of the data more visible. Additionally, the $E 1$ and $M 1 \gamma$-ray strength function curves predicted by the microscopic Gogny D1M HFB+QRPA model [59-61] have been added to Fig. 6 .

For ${ }^{64} \mathrm{Zn}$ (top panel in Fig. 6) the values obtained in this work show an overall smooth trend and no clear indication of an enhancement below the particle separation energies. Fluctuations in the data points might be because of uncertainties in the absolute branching ratios of the secondary $\gamma$ ray transitions caused by not yet observed $\gamma$-ray transitions. Overall the trend for the $\gamma$-ray strength is well predicted by the QRPA calculations.

For ${ }^{66} \mathrm{Zn}$ (bottom panel in Fig. 6), the enhancements around $7.0 \mathrm{MeV}$ are still visible and not reproduced in the
QRPA calculations. Smaller fluctuations might still be because of uncertain absolute branching ratios for secondary transitions in this analysis. However, the rise at $7.0 \mathrm{MeV}$ is mainly caused by primary intensities to $J_{f}^{\pi}=2^{+}$and $J_{f}^{\pi}=4^{+}$ states, whose branching transitions are generally well known.

At the $\gamma$-ray energy for the population of the $2_{1}^{+}$states, the $M 1$ strength predicted by the QRPA calculations is almost an order of magnitude lower than the $E 1$ strength and, thus, has little impact on the normalization. However, in the region below $9.0 \mathrm{MeV}$ the $M 1$ strength cannot be neglected anymore and might be responsible for some of the fluctuations seen in our data.

Please note that the amount of $M 1$ strength we see is determined by the ratio $(b / a)$ as described in Sec. II which might be different for other reaction types to study the $\gamma$-ray strength in the stable zinc isotopes.

\section{CONCLUSION}

To conclude, we showed that the ratios of primary $\gamma$-ray intensities in radiative proton-capture reactions can be used to study the dipole strength in the reaction product in an almost model independent way. However, the disentanglement of the $E 1$ and $M 1$ strength was shown to depend on the spin distribution, width fluctuation factors, as well as the nuclear level density in the compound nucleus and needs to be studied for each reaction and excitation energy individually. Here, we have shown, that the impact of the $M 1$ strength is quite small.

In contrast to former studies, the intensity of primary $\gamma$ rays were obtained by using coincidence techniques in $\gamma$-ray spectroscopy. Because of the high coincidence efficiency of the HORUS spectrometer, we were able to resolve many discrete secondary $\gamma$-ray transitions in two-step $\gamma$-ray spectra, which we used to obtain the intensities of the primary $\gamma$ 
TABLE III. Relative strength obtained from the primary $\gamma$-ray intensities after the scaling of strength curves for individual final spins and parities. See text for details.

\begin{tabular}{|c|c|c|c|}
\hline \multicolumn{2}{|c|}{${ }^{64} \mathrm{Zn}$} & \multicolumn{2}{|c|}{${ }^{66} \mathrm{Zn}$} \\
\hline$E_{\gamma}(\mathrm{MeV})$ & $f\left(E_{\gamma}\right)$ (a.u.) & $E_{\gamma}(\mathrm{MeV})$ & $f\left(E_{\gamma}\right)$ (a.u.) \\
\hline 11.12 & 1.911(109) & 10.82 & $1.003(128)$ \\
\hline 10.31 & $1.286(358)$ & 10.72 & $1.119(168)$ \\
\hline 10.13 & $1.000(038)$ & 9.78 & $0.966(121)$ \\
\hline 9.32 & $0.738(113)$ & 9.68 & $1.000(015)$ \\
\hline 9.32 & $0.738(091)$ & 8.95 & $0.705(089)$ \\
\hline 9.21 & $1.880(252)$ & 8.85 & $0.647(010)$ \\
\hline 8.81 & $0.795(032)$ & 8.45 & $0.379(053)$ \\
\hline 8.51 & $0.576(072)$ & 8.37 & $0.485(065)$ \\
\hline 8.51 & $0.576(111)$ & 8.35 & $0.535(012)$ \\
\hline 8.40 & $0.576(169)$ & 8.27 & $0.443(013)$ \\
\hline 8.38 & $0.495(037)$ & 8.05 & $0.371(167)$ \\
\hline 8.33 & $0.667(112)$ & 8.04 & $0.499(080)$ \\
\hline 8.11 & $0.498(062)$ & 7.96 & $0.314(014)$ \\
\hline 8.04 & $0.562(213)$ & 7.94 & $0.392(011)$ \\
\hline 8.00 & $0.324(042)$ & 7.88 & $0.560(074)$ \\
\hline 7.93 & $0.396(060)$ & 7.78 & $0.751(014)$ \\
\hline 7.86 & $0.453(072)$ & 7.74 & $0.348(054)$ \\
\hline 7.75 & $0.506(085)$ & 7.72 & $0.389(058)$ \\
\hline 7.70 & $0.515(153)$ & 7.61 & $0.564(113)$ \\
\hline 7.69 & $0.275(076)$ & 7.61 & $0.304(009)$ \\
\hline 7.57 & $0.244(169)$ & 7.59 & $0.203(033)$ \\
\hline 7.57 & $0.292(039)$ & 7.51 & $0.998(092)$ \\
\hline 7.52 & $0.346(046)$ & 7.49 & $0.409(063)$ \\
\hline 7.32 & $0.223(089)$ & 7.49 & $0.320(012)$ \\
\hline 7.23 & $0.236(060)$ & 7.39 & $0.528(113)$ \\
\hline 6.89 & $0.273(042)$ & 7.29 & $0.308(075)$ \\
\hline \multirow[t]{16}{*}{6.51} & $0.247(050)$ & 7.24 & $0.441(063)$ \\
\hline & & 7.22 & $0.218(007)$ \\
\hline & & 7.19 & $0.297(011)$ \\
\hline & & 7.15 & $0.416(131)$ \\
\hline & & 7.15 & $0.692(031)$ \\
\hline & & 7.05 & $0.956(022)$ \\
\hline & & 6.97 & $0.890(031)$ \\
\hline & & 6.93 & $0.341(024)$ \\
\hline & & 6.90 & $0.172(008)$ \\
\hline & & 6.73 & $0.265(035)$ \\
\hline & & 6.64 & $0.280(007)$ \\
\hline & & 6.52 & $0.212(048)$ \\
\hline & & 6.43 & $0.189(007)$ \\
\hline & & 6.26 & $0.213(007)$ \\
\hline & & 5.92 & $0.129(006)$ \\
\hline & & 5.87 & $0.299(016)$ \\
\hline
\end{tabular}

rays via the secondary $\gamma$ rays and their respective absolute branching ratios for the ${ }^{63,65} \mathrm{Cu}(p, \gamma \gamma)$ reactions.

Relative $\gamma$-strength function curves were calculated for transitions to final states with $J^{P}=0^{+}, 1^{+} 2^{+}, 4^{+}$and were compared to previous results. With this we were able to test the generalized Brink-Axel hypothesis in ${ }^{64} \mathrm{Zn}$ for two different excitation energies, which showed no violation.

Via a normalization, the relative values were compared to $\gamma$-ray induced experiments. A smooth trend for the dipole
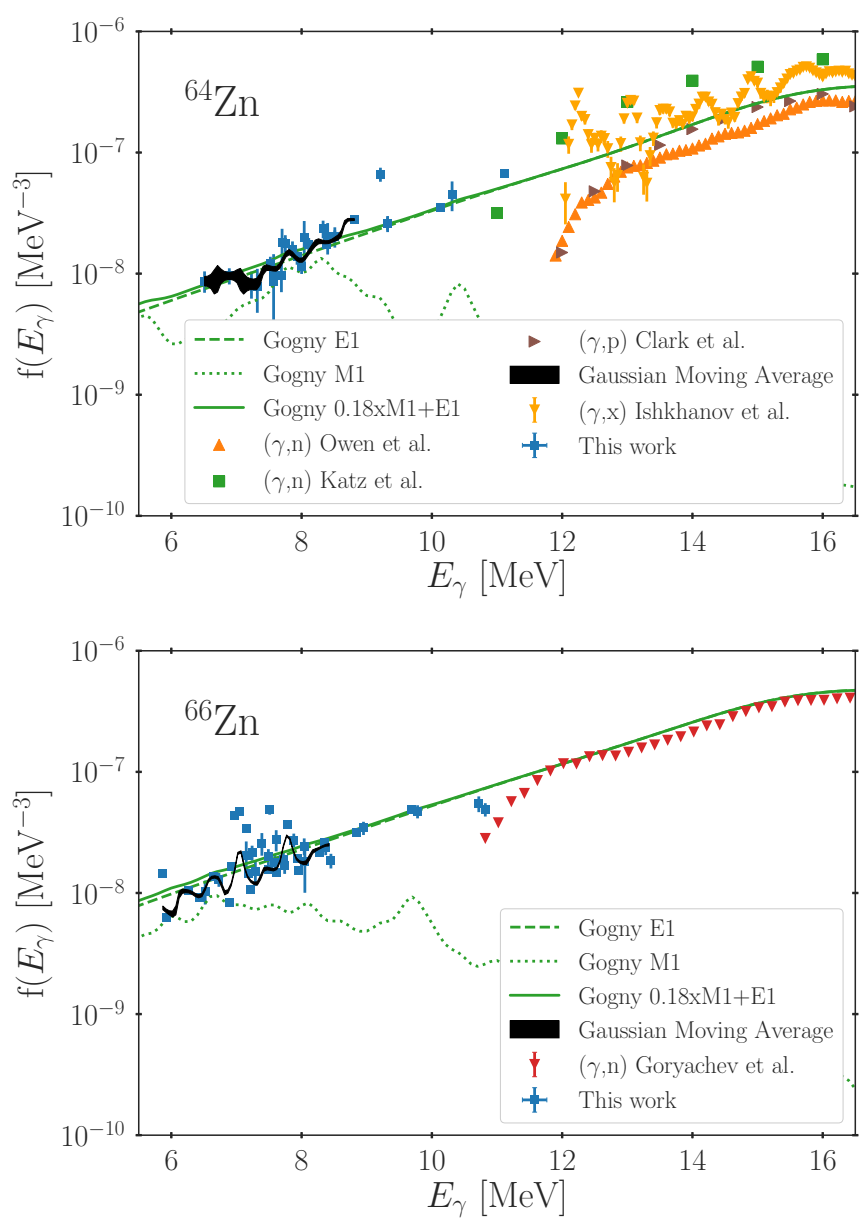

FIG. 6. Comparison of the experimentally determined $\gamma$-ray strength functions in ${ }^{64,66} \mathrm{Zn}$. The relative values obtained in this work have been normalized to the strength for the $\gamma$-ray energy of the transition to the $2_{1}^{+}$states in the zinc isotopes taken from the microscopical Gogny D1M HFB+QRPA model [59-61]. The values obtained in this work show some fluctuations which might be because of uncertainties in the absolute branching ratios caused by not yet observed $\gamma$-ray transitions. See text for details.

strength in the ${ }^{64} \mathrm{Zn}$ below the particle separation energies was observed. For ${ }^{66} \mathrm{Zn}$ a small enhancement at around 7.0 MeV is indicated by our data as well as by the data of Ref. [43]. A slightly stronger enhancement in the same energy region was recently observed in ${ }^{74} \mathrm{Zn}$ via the Oslo method [62]. Such an enhancement should be visible in precise nuclear resonance fluorescence measurements.

\section{ACKNOWLEDGMENTS}

The authors thank the accelerator staff at the Institute for Nuclear Physics in Cologne for providing excellent beams. Moreover, we gratefully acknowledge K. O. Zell and A. Blazhev for target production, H.-W. Becker, V. Foteinou, and D. Rogalla of the Ruhr-Universität Bochum for assistance during RBS measurements. This project was supported by the Deutsche Forschungsgemeinschaft under Contract 
No. ZI 510/8-1 and the ULDETIS project within the UoC Excellence Initiative institutional strategy. A.C.L. gratefully acknowledges funding from the ERC-STG-2014, under Grant
Agreement No. 637686 and A.V.V. gratefully acknowledges funding from the U.S. Department of Energy, Grant No. DENA0002905.
[1] E. M. Burbidge, G. R. Burbidge, W. A. Fowler, and F. Hoyle, Rev. Mod. Phys. 29, 547 (1957).

[2] F. Käppeler, R. Gallino, S. Bisterzo, and W. Aoki, Rev. Mod. Phys. 83, 157 (2011).

[3] M. Arnould et al., Phys. Rep. 450, 97 (2007).

[4] S. Goriely, Eur. Phys. J. A 51, 172 (2015).

[5] D. Martin et al., Astrophys. J. 813, 2 (2015).

[6] S. Goriely, Phys. Lett. B 436, 10 (1998).

[7] A. C. Larsen and S. Goriely, Phys. Rev. C 82, 014318 (2010).

[8] D. Savran et al., Prog. Part. Nucl. Phys. 70, 210 (2013).

[9] A. Voinov et al., Phys. Rev. Lett. 93, 142504 (2004).

[10] R. Schwengner, S. Frauendorf, and A. C. Larsen, Phys. Rev. Lett. 111, 232504 (2013).

[11] A. C. Larsen et al., Phys. Rev. Lett. 111, 242504 (2013).

[12] D. M. Brink, Ph.D thesis, Oxford University, Oxford, 1955.

[13] P. Axel, Phys. Rev. 126, 671 (1962).

[14] M. Guttormsen, A. C. Larsen, A. Gorgen, T. Renstrøm, S. Siem, T. G. Tornyi, and G. M. Tveten, Phys. Rev. Lett. 116, 012502 (2016).

[15] A. Schiller et al., Nucl. Instrum. Methods A 447, 498 (2000).

[16] D. Martin et al., Phys. Rev. Lett. 119, 182503 (2017).

[17] R. Schwengner et al., Phys. Rev. C 78, 064314 (2008).

[18] L. Netterdon et al., Phys. Lett. B 744, 358 (2015).

[19] H. Utsunomiya et al., Phys. Rev. C 88, 015805 (2013).

[20] J. Isaak et al., Phys. Lett. B 727, 361 (2013).

[21] J. Isaak et al., Phys. Lett. B 788, 225 (2018).

[22] S. N. Liddick et al., Phys. Rev. Lett. 116, 242502 (2016).

[23] M. Wiedeking et al., Phys. Rev. Lett. 108, 162503 (2012).

[24] F. Bečvář et al., Nucl. Instrum. Methods B 261, 930 (2007).

[25] A. M. Krumbholz et al., Phys. Lett. B 744, 7 (2015).

[26] M. D. Jones et al., Phys. Rev. C 97, 024327 (2018).

[27] M. Krtička, M. Wiedeking, F. Bečvář, and S. Valenta, Phys. Rev. C 93, 054311 (2016).

[28] S. Goriely, P. Dimitriou, M. Wiedeking et al., Eur. Phys. J. A 55, 172 (2019).

[29] F. Heim, P. Scholz, M. Körschgen, J. Mayer, M. Müller, and A. Zilges, Phys. Rev. C 101, 035805 (2020).

[30] G. A. Bartholomew, E. D. Earle, A. J. Ferguson, J. W. Knowles, and M. A. Lone, in Advances in Nuclear Physics, Vol. 7, edited by M. Baranger amd E. Vogt (Plenum Press, New York/London, 1973), Chap. 4.

[31] A. Hoogenboom, Nucl. Instrum. Methods 3, 57 (1958).

[32] S. Boneva, E. Vasileva, Y. Popov, A. Sukhovoi, and V. Khitrov, Bull. Acad. Sci. USSR, Phys. Ser. 52, 1 (1988).

[33] F. Bečvář, P. Cejnar, R. E. Chrien, and J. Kopecký, Phys. Rev. C 46, 1276 (1992).

[34] A. Voinov, S. M. Grimes, C. R. Brune, M. Guttormsen, A. C. Larsen, T. N. Massey, A. Schiller, and S. Siem, Phys. Rev. C 81, 024319 (2010).

[35] A. Voinov et al., EPJ Web Conf. 93, 01022 (2015).

[36] L. N. V. Derya, J. Endres, C. Fransen, A. Hennig, J. Mayer, C. Müller-Gatermann, A. Sauerwein, P. Scholz, M. Spieker, and A. Zilges, Nucl. Instrum. Methods A 754, 94 (2014).
[37] J. Brenneisen et al., Z. Phys. A 352, 149 (1995).

[38] J. Mayer, G4HORUS-GEANT4 Simulation for HORUS, https:// gitlab.ikp.uni-koeln.de/jmayer/g4horus.git.

[39] J. Mayer, S. Goriely, L. Netterdon, S. Péru, P. Scholz, R. Schwengner, and A. Zilges, Phys. Rev. C 93, 045809 (2016).

[40] F. Bečvář, Nucl. Instrum. Methods A 417, 434 (1998).

[41] L. E. Kirsch and L. A. Bernstein, Nucl. Instrum. Methods A 892, 30 (2018).

[42] NNDC Online Data Service, ENSDF database, https://www. nndc.bnl.gov/nndc/ensdf/.

[43] B. Erlandsson, K. Nilson, and A. Marcinkowski, Nucl. Phys., Sect. A 343, 197 (1980).

[44] L. M. Bollinger and G. E. Thomas, Phys. Rev. Lett. 18, 1143 (1967).

[45] L. M. Bollinger and G. E. Thomas, Phys. Rev. Lett 21, 233 (1968).

[46] L. M. Bollinger and G. E. Thomas, Phys. Rev. C 2, 1951 (1970).

[47] A. J. Koning, S. Hilaire, and M. C. Duijvestijn, "TALYS-1.0" in Proceedings of the International Conference on Nuclear Data for Science and Technology, April 22-27, 2007, Nice, France, edited by O. Bersillon, F. Gunsing, E. Bauge, R. Jacqmin, and S. Leray (EDP Sciences, Les Ulis, 2008), pp. 211-214.

[48] A. Koning and D. Rochman, Nucl. Data Sheets 113, 2841 (2012).

[49] J. P. Jeukenne, A. Lejeune, and C. Mahaux, Phys. Rev. C 16, 80 (1977).

[50] S. Goriely and J.-P. Delaroche, Phys. Lett. B 653, 178 (2007).

[51] A. Gilbert and A. G. W. Cameron, Can. J. Phys. 43, 1446 (1965).

[52] Y. Xu, S. Goriely, A. Jorissen, G. Chen, and M. Arnould, Astron. Astrophys. 549, A106 (2013).

[53] Y. Kalmykov, C. Özen, K. Langanke, G. Martínez-Pinedo, P. von Neumann-Cosel, and A. Richter, Phys. Rev. Lett. 99, 202502 (2007).

[54] H. M. Hofmann, J. Richert, J. W. Tepel, and H. A. Weidenmüller, Ann. Phys. 90, 403 (1975).

[55] P. A. Moldauer, Phys. Rev. C 14, 764(R) (1976).

[56] J. J. M. Verbaarschot, H. A. Weidenmüller, and M. R. Zirnbauer, Phys. Rep. 129, 367 (1985).

[57] S. Hilaire, Ch. Lagrange, and A. J. Koning, Ann. Phys. 306, 209 (2003).

[58] A. P. D. Ramirez, A. V. Voinov, S. M. Grimes, A. Schiller, C. R. Brune, T. N. Massey, and A. Salas-Bacci, Phys. Rev. C 88, 064324 (2013).

[59] M. Martini, S. Hilaire, S. Goriely, A. Koning, and S. Péru, Nucl. Data Sheets 118, 273 (2014).

[60] M. Martini, S. Péru, S. Hilaire, S. Goriely, and F. Lechaftois, Phys. Rev. C 94, 014304 (2016).

[61] S. Goriely, S. Hilaire, S. Péru, and K. Sieja, Phys. Rev. C 98, 014327 (2018).

[62] R. Lewis, S. N. Liddick, A. C. Larsen et al., Phys. Rev. C 99, 034601 (2019). 